Vol.01/ Issue. 02

Pages: 95-104

http://irojournals.com/aicn/

DOI: https://doi.org/10.36548/jaicn.2019.2.005

\title{
AN IMPROVED SAFETY ALGORITHM FOR ARTIFICIAL INTELLIGENCE ENABLED PROCESSORS IN SELF DRIVING CARS
}

\author{
Dr. Samuel Manoharan, \\ Professor, Department of Electronics, \\ Bharathiyar College Of Engineering and Technology, \\ Thiruvettakudy, Karaikal, India. \\ Email id: jsamuel@bcetedu.in
}

\begin{abstract}
The innovation in the automobiles have undergone a most noteworthy leap from the operation of the vehicles that was human centered to vehicles with the self -driving ability. The self-driving cars being a major level of attraction today, needs to concentrate also on the privacy, energy, traffic flow, environmental issues and the road safety. The perfect road safety is a goal that can be never achieved, due to the growing number of vehicles and the human population all over the world. Measures to improve the probability of the safety is put forward in the paper for the cars with the capability of the self-driving built with the artificial intelligence enabled processors. The performance evaluation of the proposed algorithm for using real time data ensures the competence of the safety algorithm in the self-driving cars.
\end{abstract}

Keywords: Self-driving Cars, Road Safety, AI Processors, Machine Learning, Safety Algorithm

\section{INTRODUCTION}

The autonomous cars with the self-driving capability are prototypically smart technology. The self-driving cars according to the author Stilgoe [1] "is never a self-driven but are driven by the social process such as the goal selection, machine making, governance use and their encounters with the world around them and autonomous is the connectivity that makes possible the driving operations to be done" Cheng [2] in his article projects the self driving cars as the ultimate aim of the intelligent vehicle development.

The self driving vehicles are capable of modifying and improving the efficiency and convince in the transportation that is based on the auto motives. Despite its capabilities it requires a proper route planning, decision making, motion planning and vehicle control in the road that is shared among the other vehicles that are either human centered or self driving human assisted vehicle or the fully self -driving cars and the pedestrians [5]. The fig.1below shows the architecture of the autonomous vehicle.

ISSN: 2582-2012 (online) 
Journal of Artificial Intelligence and Capsule Networks (2019)

Vol.01/ Issue. 02

Pages: 95-104

http://irojournals.com/aicn/

DOI: https://doi.org/10.36548/jaicn.2019.2.005

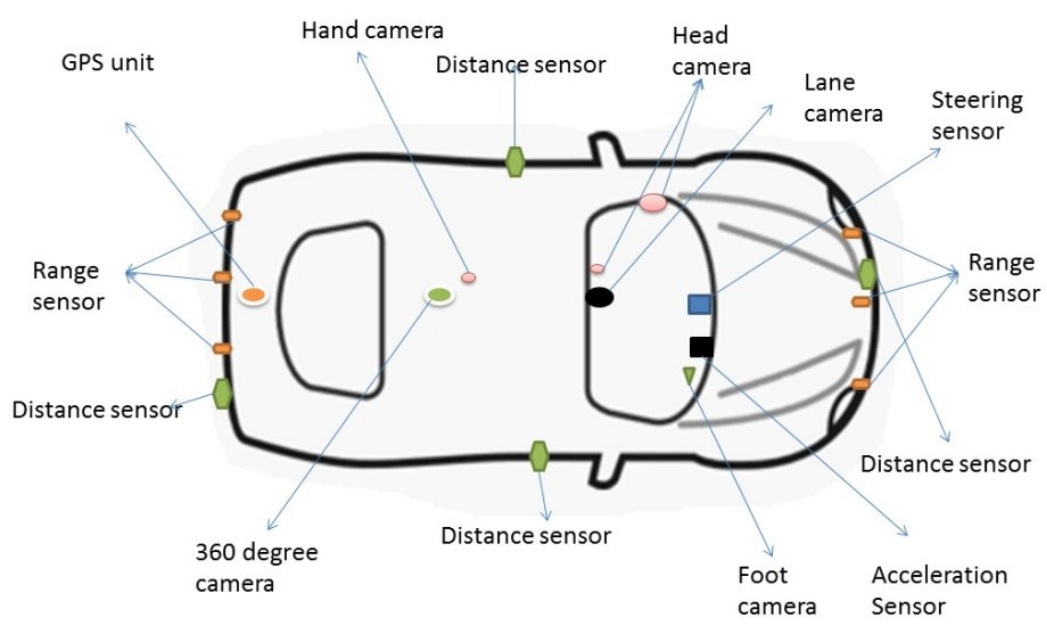

Fig.1 Architecture of the Self-Driving Car

The paper also put forward an algorithm to enhance the on road safety of the self driving cars that are operated by the artificial enabled processors with proper motion and path planning, and vehicle control.

The vehicle identification and the knowledge of the surroundings for the autonomous car in the proposed method is gained by the VANET technology [2] that was designed to assure the safety and the reliability in the transport. The VANETS using the roadside units and the vehicles as the nodes enables one to gather information about the high speed vehicle and the congestion on the road, the status of the traffic light, the application of sudden brake and change of lane by the nearby vehicles.

Artificial intelligence enabled processor (2nd generation XEON scalable processor) ensures a proper data collection in the self driving cars, providing a quick deployment, high and deterministic performance, along with the efficiency that is incredible in terms of application, controlling, packet processing and signal processing.

The remaining of the paper is arranged with the related works in the section 2 , the proposed safety algorithm in the section 3 and the results and discussion in section 4 and the conclusion in section 5 . 
Journal of Artificial Intelligence and Capsule Networks (2019)

Vol.01/ Issue.02

Pages: 95-104

http://irojournals.com/aicn/

DOI: https://doi.org/10.36548/jaicn.2019.2.005

\section{RELATED WORKS}

The article by Stilgoe, et al [1] includes the "public debate about the innovation found in the selfdriving cars , taking into consideration the competing definitions of the problems, the solutions for it, the concerns, the politics in the machine learning and the algorithms framed and the outcomes attained.

Cheng et al [2] the author puts forth an " $5 \mathrm{G}$ enabled intelligent vehicular frame work that is co-operative, to enhances the performance of the vehicles by enabling a data processing capability utilizing the vehicular network. The author aims in developing wireless system architecture for the self driving utilizing the $5 \mathrm{G}$ networks.

Bertozzi, et al [3] the paper is the" survey on the most common approaches to the autonomous road development that has been carried out worldwide, it also describes the motivations that underlie the development of the vision based intelligent vehicle along with the perspectives in the evolution of the intelligent vehicles"

Levinson et al [4] the paper presents the overview algorithms to improve the driving system of the autonomous vehicle in the urban areas, which has an unpredictable traffic, the disasters caused due to the natural calamities and the challenges faced on road in real time.

Paden et al [5] the paper aims in "developing the survey on the current state of art planning and the algorithms to control the self driving cars in the urban areas. The approaches surveyed are categorized based on the vehicle mobility, the model used, environmental frame work, and the computational requirements".

Wei, et al [6] the paper presents the "autonomous driving research with the a broader range of autonomous and intelligent behaviors that include a smooth and the convenient trajectory generation, lane keeping, lane changing, and the intersection handling either in the presence or the absence of the four wheelers, two wheelers and the pedestrians.

González et al [7] the paper provides the "review of the motion planning techniques that are utilized in the intelligent vehicles. The main aim of the paper is to focus on the computing approaches such as the enhancing safety, convenience, and the energy optimization.

ISSN: 2582-2012 (online) 
Journal of Artificial Intelligence and Capsule Networks (2019)

Vol.01/ Issue. 02

Pages: 95-104

http://irojournals.com/aicn/

DOI: https://doi.org/10.36548/jaicn.2019.2.005

$\mathrm{Li}$, et al [8] the work proceeded in the paper "develops a safety warning, and the driver assistance along with the automatic pilot for the Spring robot-autonomous vehicle in the rural and the urban areas, the proffered system utilizes multiple sensors and the digital mapping followed by the framing and describing of various lane marking detection algorithm".

Meiring, et al [9] the author puts forward the analysis of the various driving styles to examine the machine learning and the artificial intelligence algorithms involved in the behavior of the driver, the style followed in driving, the identification of the driver, driver safety and the identification of the road accidents.

Li et al [10] the "paper puts forward the real-time trajectory planning for the autonomous vehicles driving in the realistic urban environments. The paper follows a hierarchical motion planning utilizing the conjugate gradient nonlinear optimization algorithm and the cubic B-spine curve algorithm.

$\mathrm{Xu}$ et al [11] the paper proposes a motion planning frame work of the autonomous vehicles for the uncertain traffic conditions that are prevailing on road utilizing the Gaussian propagation and the linear quadratic Gaussian.

Magdici et al [12] the paper put forward a fail-safe motion planner for generating the optimal trajectory that guarantees safety at all times

\section{PROPOSED WORK}

The driverless cars also known as the self driving cars do not require any human drivers to perform the operation such as the steering or pedaling etc. They are capable of perceiving the surroundings that is shared by the other vehicles and pedestrians along with the traffic light signals, pot holes, contours and the disasters caused in the road. The mains features that work in pipeline to make the self-driving cars possible is listed as follows.

1. Computer Vision - The main forward cameras, covering a maximum distance of the $150 \mathrm{~m}$, the rearward looking cameras for maximum distance of $100 \mathrm{~m}$, the wide forward cameras for a distance of $60 \mathrm{~m}$, ultrasonic, forward looking side cameras, RADAR and the LIDAR enables the collection and interpretation of the data that are sensed.

2. Sensor fusion: the information's gathered are put together to decide the future action to be taken.

3. Localization - enabled by the GPS module in the car. 
Journal of Artificial Intelligence and Capsule Networks (2019)

Vol.01/ Issue.02

Pages: 95-104

http://irojournals.com/aicn/

DOI: https://doi.org/10.36548/jaicn.2019.2.005

4. Path planning - deciding the proper path avoiding the collision and obstacle.

5. Control- it includes the decision such as the emergency stop controlling the steering, brake and the clutch of the car.

The block diagram in fig .2 shows the working of the self driving car that is enabled with the AI processor.

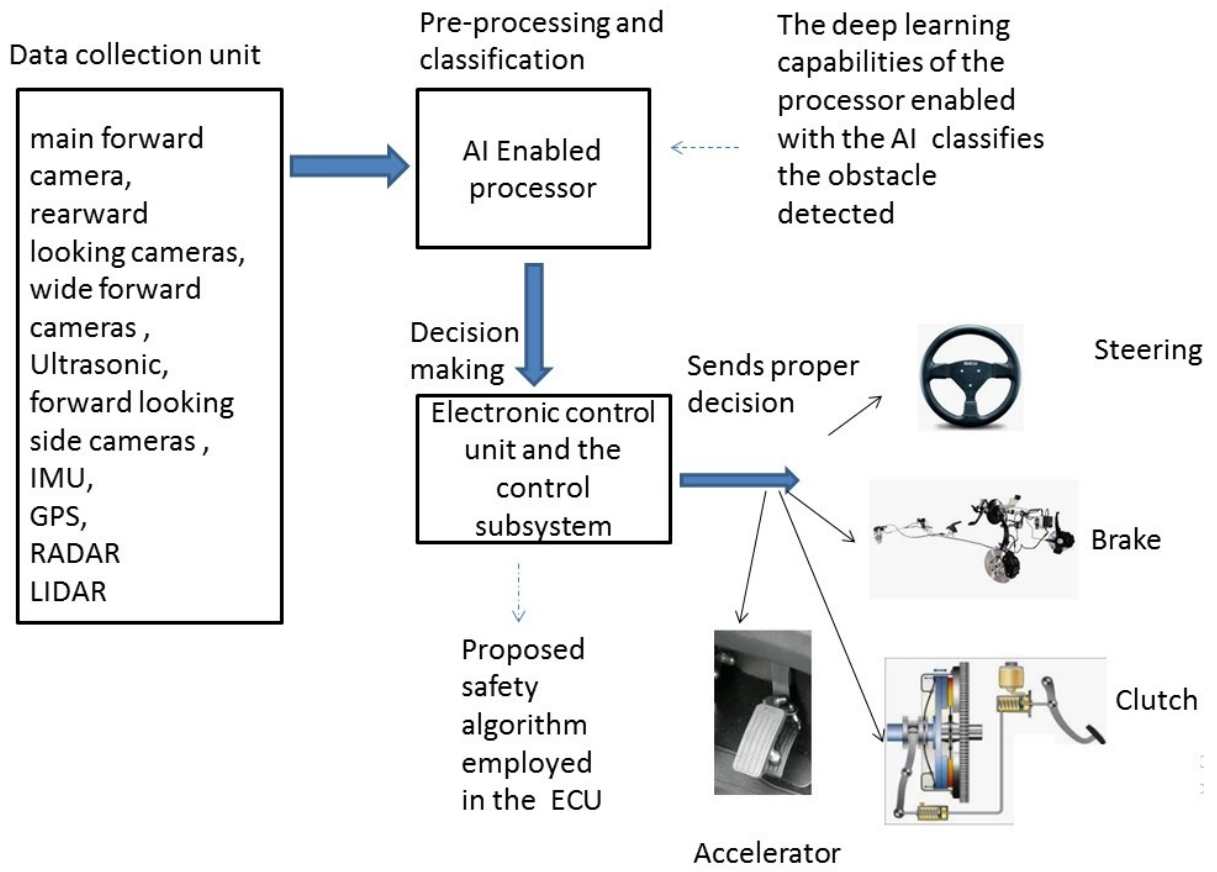

Fig .2. Working of the Self Driving Cars

The computer vision enabled in the self driving cars enable them to see all the happenings around, using the mono, stereo cameras, RADAR and the LIDAR sensor fitted to it. This behaves as the eye to the driverless car the information sensed are fused together and preprocessed and classified utilizing the 2nd generation XEON scalable processor that is enabled with the deep learning processes to classify the obstacles detected by the sensors and the cameras. The classified information is forwarded to the Electronic control unit and the control subsystem that controls the entire automotive units in the car such as the engine, brakes, doors, windows, clutch and the accelerator. 
Journal of Artificial Intelligence and Capsule Networks (2019)

Vol.01/ Issue.02

Pages: 95-104

http://irojournals.com/aicn/

DOI: https://doi.org/10.36548/jaicn.2019.2.005

As the paper concentrates on the improved safety of the self driving cars it put forwards the algorithm that ensure an enhanced decision making in the self-driving cars, providing a proper trajectory adjustment on detection of the obstacle such as the pedestrians, traffic lights, and other vehicles.

\subsection{PROPOSED ALGORITHM FOR TRAJECTORY ADJUSTMENT TO IMPROVE SAFETY}

As the proposed method concentrates on the improving the safety utilizing the proper trajectory planning avoiding the collisions on the obstacles and the perfect decision to be taken on when coming across a pedestrian or the traffic light signal. The distance sensor engaged in the cars enables us to identify the distance between the vehicles that are sharing the road with the self-driving cars. The proposed method incorporates the conjugate nonlinear optimization put forward by the Li [10] and the Linear -quadratic Gaussian propagation put forward by the Xu, [11] (CNO-LQP)to improve the road safety by providing a perfect trajectory optimization and proper motion planning and vehicle control. The fig .3 shows the obstacle avoidance by the self-driving car.

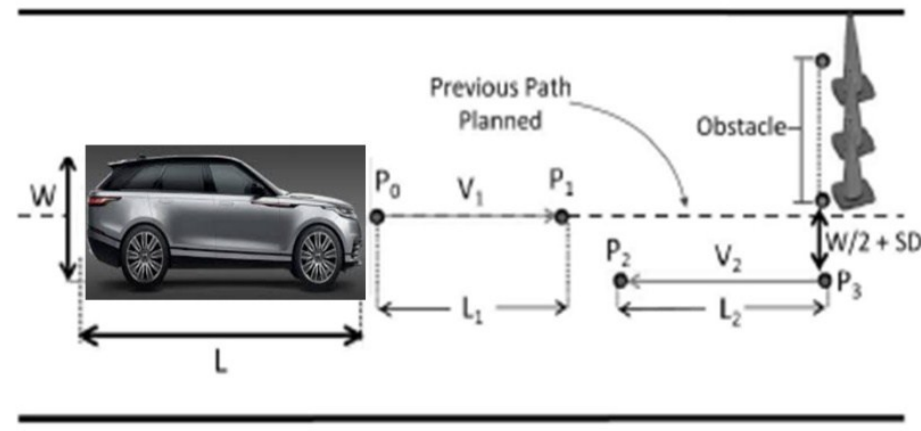

Fig.3 Obstacle Avoidance [11]

The conjugate nonlinear optimization smoothens and interpolates the available paths and a proper trajectory planning, and the Linear -quadratic Gaussian propagation handles the vehicle control to be initiated on detecting the obstacle. The flow chart below in fig. 4 shows the path planning and the control initiated by the self driving car on detecting the obstacle. 
Journal of Artificial Intelligence and Capsule Networks (2019)

Vol.01/ Issue.02

Pages: 95-104

http://irojournals.com/aicn/

DOI: https://doi.org/10.36548/jaicn.2019.2.005

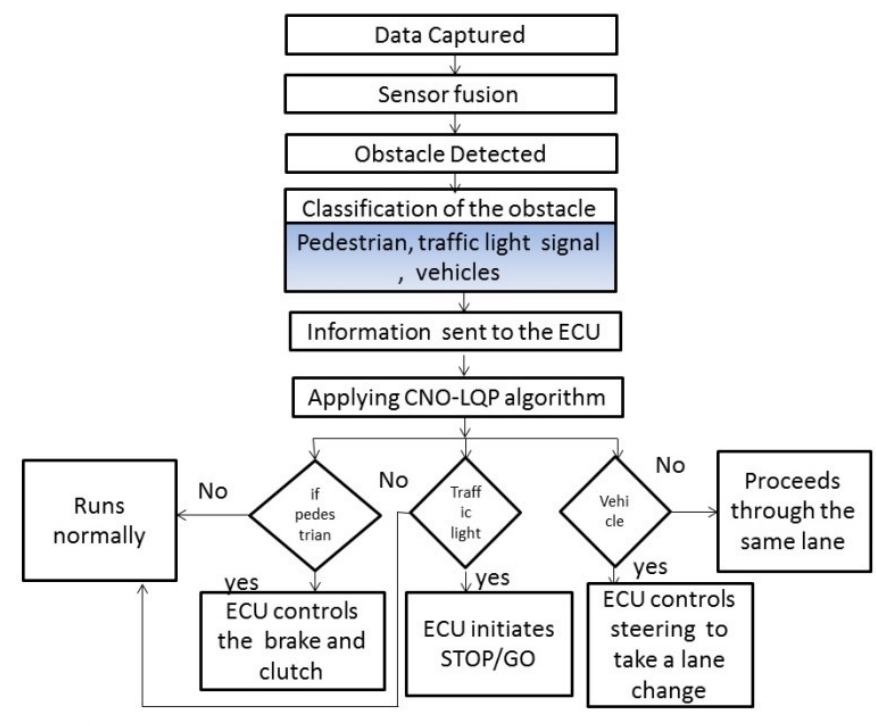

Fig.4 Flow Chart of Path Planning and Vehicle Control

\section{RESULTS AND DISCUSSION}

The safety algorithm proposed incorporating the conjugate nonlinear optimization and the Linear -quadratic Gaussian propagation is tested with the real time traffic scenario. Ensures the improved safety provided for the self driving cars in the lane changing, the obstacle detection and the vehicle control. The fig .5 below shows the snap shots of the self driving cars implemented with the improved safety algorithm with a proper planning and the vehicle control.

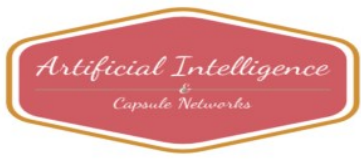


Journal of Artificial Intelligence and Capsule Networks (2019)

Vol.01/ Issue. 02

Pages: 95-104

http://irojournals.com/aicn/

DOI: https://doi.org/10.36548/jaicn.2019.2.005

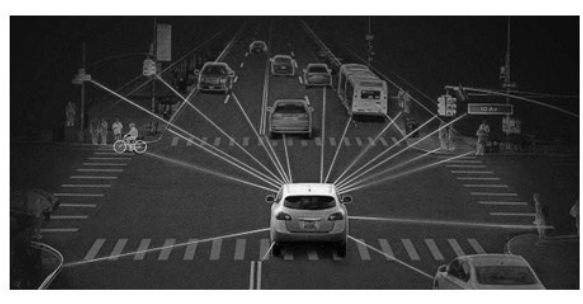

(a)

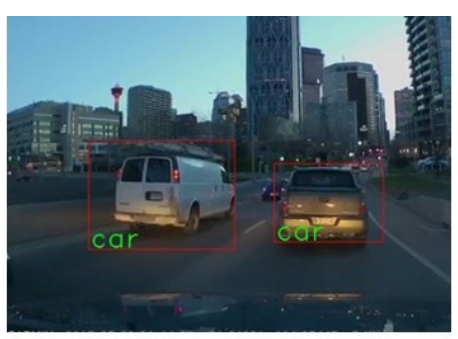

(b)
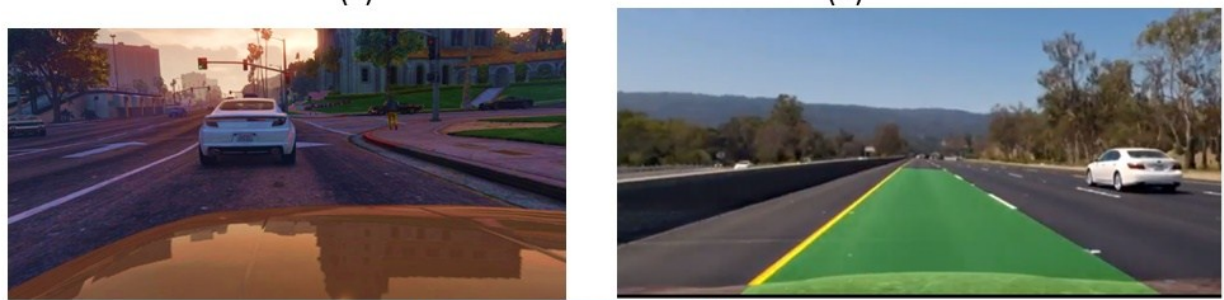

(c)

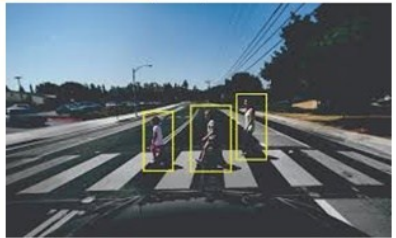

(d)

(e)

Fig.5.The Evaluation of the Proposed in the Real Time Scenario (a) capturing of information, (b) classification of the obstacle detected (c) Response of the self-driving car for the traffic signal (d) Trajectory planning (e) Response to the pedestrians

The above fig .5 shows the response of the self driving vehicles in the different real time scenarios, thus making the transport safer for the both the passengers in and out of the vehicle.

\subsection{Advantages of the self-driving car include:}

Improved safety- drastically reducing fatalities

Reduced carbon emission - due to the elimination of frequent braking and accelerating Independent of human intervention - eludes the necessity of the human drivers

Less road congestion - the programming in the self driving allows space for more than two or three cars to smoothly change lane in the highways.

Apart from this they also provide an increased productivity, self-parking capability, potentially higher speed limits.

ISSN: 2582-2012 (online) 
Journal of Artificial Intelligence and Capsule Networks (2019)

Vol.01/ Issue.02

Pages: 95-104

http://irojournals.com/aicn/

DOI: https://doi.org/10.36548/jaicn.2019.2.005

5. CONCLUSION

The self driving cars would change the way of living, working and playing creating a safer environment that is pollution free. The paper presenting the safety algorithm that enables the driverless car to make a proper decision on its motion planning, path planning and the vehicle control by providing a perfect trajectory adjustment and decision making on the obstacle detection, ensures a highly efficient and a safer driving on road that is shared by the other vehicles and the pedestrians. The use of the driver less cars on the road due the timely and the proper decision would avoid the unnecessary congestion on road and the occurrence of the fatalities paving way for a safer environment.

\section{References}

[1] Stilgoe, Jack. "Machine learning, social learning and the governance of self-driving cars." Social studies of science 48, no. 1 (2018): 25-56.

[2] Cheng, Xiang, Chen Chen, Wuxiong Zhang, and Yang Yang. "5G-enabled cooperative intelligent vehicular (5GenCIV) framework: When Benz meets Marconi." IEEE Intelligent Systems 32, no. 3 (2017): 53-59.

[3] Bertozzi, Massimo, Alberto Broggi, and Alessandra Fascioli. "Vision-based intelligent vehicles: State of the art and perspectives." Robotics and Autonomous systems 32, no. 1 (2000): 1-16.

[4] Levinson, Jesse, Jake Askeland, Jan Becker, Jennifer Dolson, David Held, Soeren Kammel, J. Zico Kolter et al. "Towards fully autonomous driving: Systems and algorithms." In 2011 IEEE Intelligent Vehicles Symposium (IV), pp. 163-168. IEEE, 2011.

[5] Paden, Brian, Michal Čáp, Sze Zheng Yong, Dmitry Yershov, and Emilio Frazzoli. "A survey of motion planning and control techniques for self-driving urban vehicles." IEEE Transactions on intelligent vehicles 1, no. 1 (2016): 33-55.

[6] Wei, Junqing, Jarrod M. Snider, Junsung Kim, John M. Dolan, Raj Rajkumar, and Bakhtiar Litkouhi. "Towards a viable autonomous driving research platform." In 2013 IEEE Intelligent Vehicles Symposium (IV), pp. 763-770. IEEE, 2013.

[7] González, David, Joshué Pérez, Vicente Milanés, and Fawzi Nashashibi. "A review of motion planning techniques for automated vehicles." IEEE Transactions on Intelligent Transportation Systems 17, no. 4 (2015): 1135-1145.

[8] Li, Qing, Nanning Zheng, and Hong Cheng. "Springrobot: A prototype autonomous vehicle and its algorithms for lane detection." IEEE Transactions on Intelligent Transportation Systems 5, no. 4 (2004): 300-308.

[9] Meiring, Gys, and Hermanus Myburgh. "A review of intelligent driving style analysis systems and related artificial intelligence algorithms." Sensors 15, no. 12 (2015): 30653-30682. 
Journal of Artificial Intelligence and Capsule Networks (2019)

Vol.01/ Issue. 02

Pages: 95-104

http://irojournals.com/aicn/

DOI: https://doi.org/10.36548/jaicn.2019.2.005

[10] Li, Xiaohui, Zhenping Sun, Dongpu Cao, Zhen He, and Qi Zhu. "Real-time trajectory planning for autonomous urban driving: Framework, algorithms, and verifications." IEEE/ASME Transactions on Mechatronics 21, no. 2 (2015): 740-753.

[11] Xu, Wenda, Jia Pan, Junqing Wei, and John M. Dolan. "Motion planning under uncertainty for on-road autonomous driving." In 2014 IEEE International Conference on Robotics and Automation (ICRA), pp. 2507-2512. IEEE, 2014.

[12] Magdici, Silvia, and Matthias Althoff. "Fail-safe motion planning of autonomous vehicles." In 2016 IEEE 19th International Conference on Intelligent Transportation Systems (ITSC), pp. 452-458. IEEE, 2016. 